\title{
Association between Metformin Use and Cancer Stage at Diagnosis among Elderly Medicare Beneficiaries with Preexisting Type 2 Diabetes Mellitus and Incident Prostate Cancer
}

\author{
Amit D. Raval, ${ }^{1,2}$ Malcolm D. Mattes, ${ }^{3}$ Suresh Madhavan, ${ }^{1}$ Xiaoyun Pan, ${ }^{4}$ \\ Wenhui Wei, ${ }^{5}$ and Usha Sambamoorthi ${ }^{1}$ \\ ${ }^{1}$ Department of Pharmaceutical Systems and Policy, School of Pharmacy, West Virginia University, Morgantown, WV 26505, USA \\ ${ }^{2}$ Healthcore, Inc., Wilmington, DE 19801, USA \\ ${ }^{3}$ Department of Radiation Oncology, School of Medicine, West Virginia University, Morgantown, WV 26505, USA \\ ${ }^{4}$ Evidera, Lexington, MA 02420, USA \\ ${ }^{5}$ Sanofi U.S., Inc., Bridgewater, NJ 08807, USA
}

Correspondence should be addressed to Amit D. Raval; amitravalwaves@gmail.com

Received 5 December 2015; Accepted 9 May 2016

Academic Editor: Daniela Foti

Copyright (C) 2016 Amit D. Raval et al. This is an open access article distributed under the Creative Commons Attribution License, which permits unrestricted use, distribution, and reproduction in any medium, provided the original work is properly cited.

Objective. To examine the association between metformin use and cancer stage at diagnosis among elderly men with preexisting diabetes mellitus and incident prostate cancer. Methods. This study used a population-based observational cohort of elderly men ( $\geq 66$ years) with preexisting diabetes and incident prostate cancer between 2008 and $2009(N=2,652)$. Cancer stage at diagnosis (localized versus advanced) was based on the American Joint Cancer Committee classification. Metformin use and other independent variables were measured during the one year before cancer diagnosis. Logistic regressions with inverse probability treatment weights were used to control for the observed selection bias. Results. A significantly lower percentage of metformin users were diagnosed with advanced prostate cancer as compared to nonusers ( $4.7 \%$ versus $6.7 \%, p<0.03)$. After adjusting for the observed selection bias and other independent variables, metformin use was associated with a $32 \%$ reduction in the risk of advanced prostate cancer (adjusted odds ratio, AOR: 0.68, 95\% confidence interval, CI: 0.48, 0.97). Conclusions. This is the first epidemiological study to support the role of metformin in reducing the risk of advanced prostate cancer. Randomized clinical trials are needed to confirm the causal link between metformin use and prostate cancer diagnosis stage.

\section{Introduction}

Individuals with diabetes, specifically those with type 2 diabetes mellitus (T2DM) have a higher risk for many cancers such as the breast, colon and rectum, endometrium, liver, and pancreatic cancers as compared to those without diabetes [1] due to biological mechanisms and shared risk factors [2-4]. In preclinical studies, T2DM has been found to be associated with increased levels of plasma insulin, insulin resistance, and hyperglycemia, which may have a direct effect on the growth of tumors $[5,6]$ leading to the development of many types of cancers [1].
In the case of prostate cancer, an inverse relationship between diabetes and cancer risk has been observed [3]. However, among men who developed prostate cancer, diabetes was associated with an advanced stage of cancer diagnosis $[7,8]$. Three population-based studies in the United States (US) reported that the presence of diabetes was associated with an increased risk for advanced prostate cancer measured either by stage or tumor grade. In case-control studies and cohort studies, men with diabetes were less likely to be diagnosed with localized stage of prostate cancer. The risk ratio (RR) was $0.70,95 \% \mathrm{CI}=0.56-0.86$ for case-control 
studies, and the RR was $0.72,95 \%$ CI $0.67,0.77$ for cohort studies [7]. Although the exact biological mechanisms for the link between diabetes and prostate cancer are not known, it is speculated that men with diabetes have low levels of androgen, which may be associated with advanced stage of cancer at diagnosis $[7,8]$.

The main modality of treatment for diabetes is pharmacotherapy with antidiabetes drugs such as metformin, thiazolidinediones, sulfonylureas, and insulin. All classes of antidiabetes drugs may indirectly affect the risk of prostate cancer by controlling hyperglycemia. Of special interest is the use of metformin for diabetes management because of its unique actions on insulin resistance and hyperinsulinemia [9] as well as its anticancer properties [10-12]. A systemic review found that metformin use reduced the risk of prostate cancer among men with diabetes [13] perhaps by regulating adenosine monophosphate-activated protein kinase (AMPK) pathways [10] and mammalian target of rapamycin (mTOR) [12]. Based on preclinical evidence, one can speculate that metformin may also reduce the risk of advanced prostate cancer among men with diabetes and incident prostate cancer [14]. This relationship between metformin and advanced prostate cancer diagnosis was explored by one population-based study from Canada [15]. The study investigators used a cohort of 119,315 men with diabetes and measured cancer stage using tumor grade. After adjusting for other risk factors, the investigators concluded that metformin use was not associated with the advanced form of cancer diagnosis [15]. The investigators of the study did not control for severity of diabetes, which may have affected the findings. Controlling for diabetes severity is important because individuals with severe diabetes have micro- and macrovascular complications and may have adverse pathological profiles [16]. Therefore, diabetes severity may alter the relationship between metformin use and cancer stage at diagnosis. Furthermore, the study did not control for the observed selection bias between metformin users and nonusers; such bias may lead to misleading findings on the association between metformin use and cancer stage at diagnosis. Thus, there is a need for population-based studies to examine the relationship between metformin use and cancer stage at diagnosis that control for a comprehensive set of risk factors and the observed selection bias between metformin users and nonusers.

Therefore, the current study is conducted to investigate the association between metformin use and cancer stage at diagnosis among elderly fee-for-service Medicare beneficiaries with T2DM and incident prostate cancer.

\section{Methods}

2.1. Study Design. A cohort study design was adopted with a baseline and an index date as depicted in Figure 1. The index date was defined as the date of diagnosis of prostate cancer. The baseline period consisted of 12 months before the index date. Diabetes, metformin use, and other independent variables were identified during the baseline period. The types of initial cancer treatment were identified during the followup period.

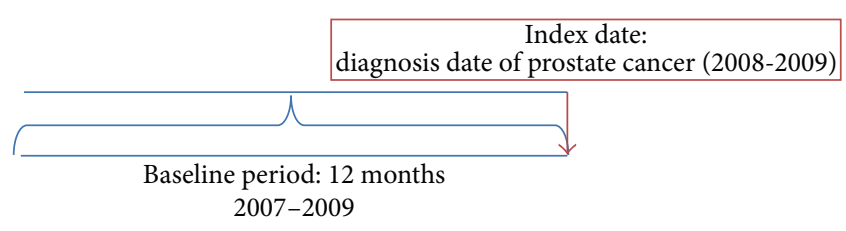

FIGURE 1: Schematic presentation of study design to examine the relationship between metformin use and cancer stage at diagnosis.

2.2. Data Source. Data were derived from the SEER-Medicare linked database. The SEER data comprised 18 populationbased cancer registries having precise and accurate information on all newly diagnosed cancer cases since 1973. At present, the data consisted of a total of 1,847,363 cases of all cancers and 340,769 cases of prostate cancer among the elderly population whose age was at least 65 at the time of diagnosis of cancer presenting. With $98 \%$ ascertainment of cases with the medical records and the highest level of certification of data quality from the North American Association of Central Cancer Registries, the SEER data are considered to be the most comprehensive and highquality population-based data on cancer incidence and their treatment and outcomes. The Patient Entitlement and Diagnosis Summary File (PEDSF) provides information on the cancer diagnosis up to ten cancers, types of cancers, cancer stage, individual's demographic attributes, marital status, and tumor characteristics at the time of cancer diagnosis. The Medicare is the primary health insurer for $97 \%$ of the US population aged 65 years and older [17]. A total of 93\% of men aged 65 years and older in SEER have been linked to Medicare population enrollment records [18]. The Medicare part of the database is comprised of the Medicare Provider Analysis and Review (MEDPAR) files, the carrier claims (old name, physician/supplier $(\mathrm{NCH})$ ), outpatient (OUTPT), and Part D Event (PDE). The MEDPAR and outpatient file include Medicare Part A claims records from any short term or long term hospital or skilled nursing facility stay from each calendar year while the outpatient file had Part B claims with outpatient visits. Each record represents an episode of hospital stay and has up to 10 diagnoses according to the International Coding of Diseases, 9th EditionCode Modification (ICD-9-CM) and 10 ICD-9-CM procedures during each stay, on the day of admission, and on the day of discharge. The carrier file represents billing records from physicians and noninstitutional providers and has procedure codes according tothe Health Care Procedure Classification Code (HCPCS) and the Common Procedural Terminology, 4th Edition (CPT-4) and ICD-9-CM procedure codes with service dates [19]. Medicare introduced optional/volunteer Part D plans in 2006 that cover prescription drugs benefit through enrollment in a Medicare advantage prescription drug plan (MA-PD) or a stand-alone drug plan (PDP). Almost half of the Medicare Parts A and B enrollee were also enrolled in Part D plans [20]. We utilized the data of the Medicare Part D Event (PDE) file in addition to PEDSF, MEDPAR, OUTPT, and NCH for the years 2007 to 2010. We examined prostate cancer cases diagnosed between 2008 and 


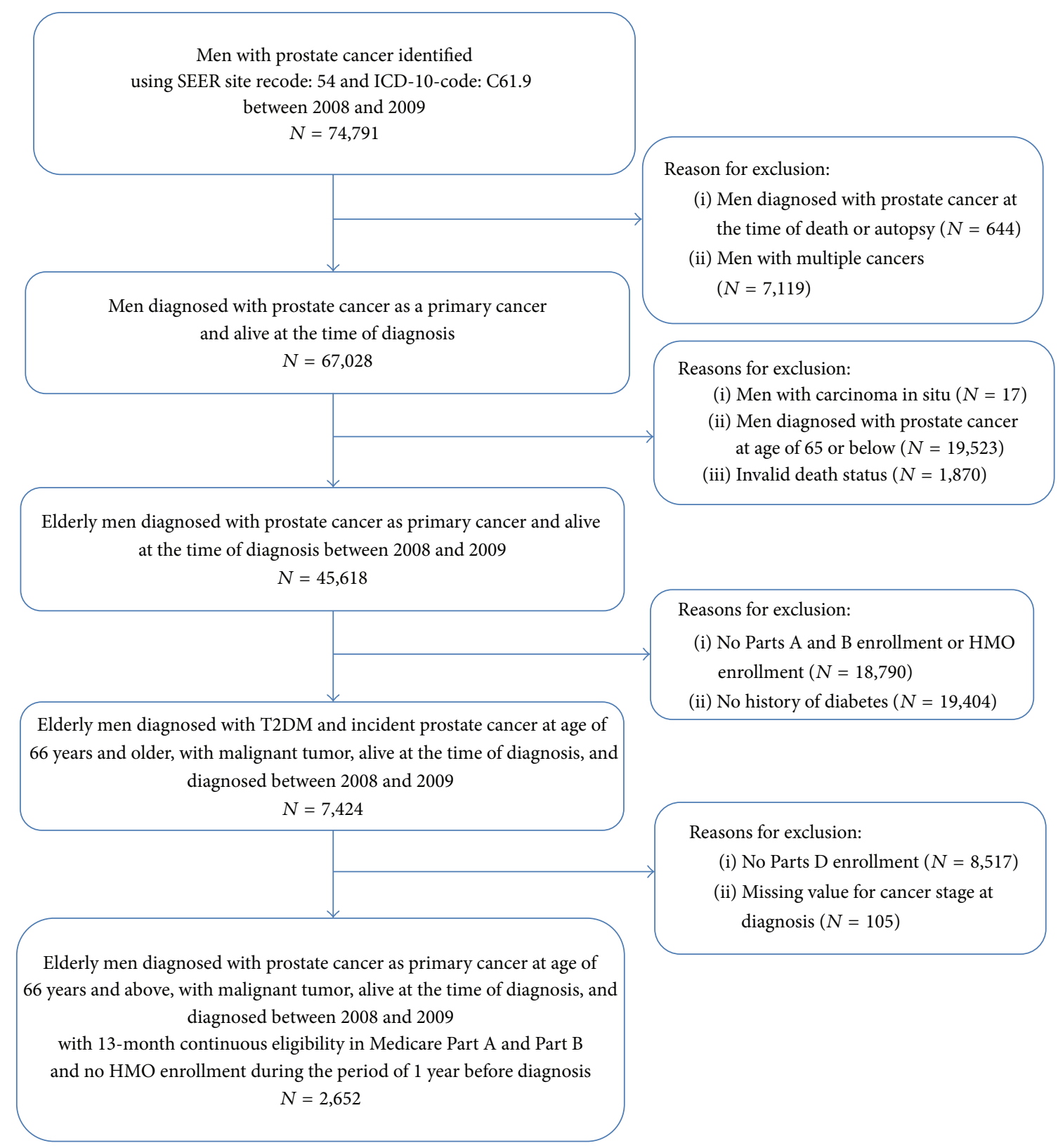

FIGURE 2: Study cohort development flow diagram for study population of elderly Medicare beneficiaries diagnosed with prostate cancer and diabetes.

2009 so that we could study their medication status in a year prior to diagnosis of cancer.

2.3. Study Cohort. The study cohort comprised 45,618 men with incident prostate cancer diagnosed between 2008 and 2009. We excluded 42,966 cases for the following reasons: those diagnosed with prostate cancer during the autopsy; those who had multiple cancers; those who had carcinoma in situ; those aged 65 years and younger; those who died during the study period; those enrolled in Medicare Health Maintenance Organizations; those not continuously enrolled in Medicare Parts A, B, and D during the study period, and those missing cancer stage at diagnosis. The details on the study cohort selection process are provided in Figure 2.

After all the exclusions, the final study cohort consisted of 2,652 elderly men with type 2 diabetes mellitus (T2DM) and incident prostate cancer. To note, every year nearly half of enrolled Medicare beneficiaries in Parts A and B are also enrolled in Medicare Part D since 2006. Therefore, our study population with prostate cancer and T2DM reduced to 7,424 enrolled in Medicare Parts A and B to 2,652 with enrollment Part D. To reduce the selection bias, we compared the characteristics of elderly men with T2DM and incident prostate cancer enrolled in Medicare Parts A and B to those 
TABLE 1: Characteristics of the study cohort elderly Medicare beneficiaries with diabetes and incident prostate cancer by metformin use SEER-Medicare linked database, 2007-2010.

\begin{tabular}{|c|c|c|c|c|c|c|c|}
\hline \multirow{3}{*}{ All } & \multicolumn{2}{|c|}{ Overall } & \multicolumn{2}{|c|}{ Metformin users } & \multicolumn{2}{|c|}{ Non-metformin-user } & \multirow{3}{*}{ Sig. } \\
\hline & $N$ & $\%$ & $N$ & $\%$ & $N$ & $\%$ & \\
\hline & 2,652 & 100 & 948 & 35.7 & 1,704 & 64.3 & \\
\hline \multicolumn{8}{|l|}{ Predisposing characteristics } \\
\hline Age at diagnosis, in years & & & & & & & $* * *$ \\
\hline $66-74$ & 1,579 & 59.5 & 609 & 64.2 & 970 & 56.9 & \\
\hline $75+$ & 1,073 & 40.5 & 339 & 35.8 & 734 & 43.1 & \\
\hline Race/ethnicity & & & & & & & * \\
\hline Whites & 1,914 & 72.2 & 680 & 71.7 & 1,234 & 72.4 & \\
\hline African American & 323 & 12.2 & 108 & 11.4 & 215 & 12.6 & \\
\hline Hispanic/Latino & 150 & 5.7 & 70 & 7.4 & 80 & 4.7 & \\
\hline Others & 265 & 10.0 & 90 & 9.5 & 175 & 10.3 & \\
\hline \multicolumn{8}{|l|}{ Marital status } \\
\hline Unmarried & 222 & 8.4 & 73 & 7.7 & 149 & 8.7 & \\
\hline Married & 1,550 & 58.4 & 581 & 61.3 & 969 & 56.9 & \\
\hline Divorced/separated & 393 & 14.8 & 136 & 14.3 & 257 & 15.1 & \\
\hline Others & 487 & 18.4 & 158 & 16.7 & 329 & 19.3 & \\
\hline \multicolumn{8}{|l|}{ Enabling characteristics } \\
\hline Quartile of median census of 2000 income & & & & & & & * \\
\hline$\$ 7-\$ 34,522$ & 664 & 25.0 & 265 & 28.0 & 399 & 23.4 & \\
\hline$\$ 34,523-46,224$ & 664 & 25.0 & 243 & 25.6 & 421 & 24.7 & \\
\hline$\$ 46,229-62,764$ & 664 & 25.0 & 229 & 24.2 & 435 & 25.5 & \\
\hline$\$ 62,767-200,008$ & 660 & 24.9 & 211 & 22.3 & 449 & 26.3 & \\
\hline \multicolumn{8}{|l|}{ Quartile of median census of 2000 education } \\
\hline $0-8.52$ & 666 & 25.1 & 222 & 23.4 & 444 & 26.1 & \\
\hline $8.53-15.16$ & 655 & 24.7 & 229 & 24.2 & 426 & 25.0 & \\
\hline $15.17-26.09$ & 664 & 25.0 & 248 & 26.2 & 416 & 24.4 & \\
\hline $26.1-100$ & 667 & 25.2 & 249 & 26.3 & 418 & 24.5 & \\
\hline \multicolumn{8}{|l|}{ PSA screening } \\
\hline Yes & 2,415 & 91.1 & 872 & 92.0 & 1,543 & 90.6 & \\
\hline No & 237 & 8.9 & 76 & 8.0 & 161 & 9.4 & \\
\hline \multicolumn{8}{|l|}{ Visit to a $P C P$} \\
\hline Yes & 1,858 & 70.1 & 672 & 70.9 & 1,186 & 69.6 & \\
\hline No & 794 & 29.9 & 276 & 29.1 & 518 & 30.4 & \\
\hline Statin use & & & & & & & $* * *$ \\
\hline Yes & 1,576 & 59.4 & 656 & 69.2 & 920 & 54.0 & \\
\hline No & 1,076 & 40.6 & 292 & 30.8 & 784 & 46.0 & \\
\hline Insulin & & & & & & & $* * *$ \\
\hline Yes & 102 & 3.8 & 62 & 6.5 & 40 & 2.3 & \\
\hline No & 2,550 & 96.2 & 886 & 93.5 & 1,664 & 97.7 & \\
\hline \multicolumn{8}{|l|}{ External environment characteristics } \\
\hline SEER-regions & & & & & & & $* *$ \\
\hline Northeast & 487 & 18.4 & 146 & 15.4 & 341 & 20.0 & \\
\hline South & 570 & 21.5 & 223 & 23.5 & 347 & 20.4 & \\
\hline North-central & 351 & 13.2 & 112 & 11.8 & 239 & 14.0 & \\
\hline West & 1,244 & 46.9 & 467 & 49.3 & 777 & 45.6 & \\
\hline
\end{tabular}


TABLE 1: Continued.

\begin{tabular}{|c|c|c|c|c|c|c|c|}
\hline \multirow{3}{*}{ All } & \multicolumn{2}{|c|}{ Overall } & \multicolumn{2}{|c|}{ Metformin users } & \multicolumn{2}{|c|}{ Non-metformin-user } & \multirow{3}{*}{ Sig. } \\
\hline & $N$ & $\%$ & $N$ & $\%$ & $N$ & $\%$ & \\
\hline & 2,652 & 100 & 948 & 35.7 & 1,704 & 64.3 & \\
\hline \multicolumn{8}{|c|}{ Need characteristics } \\
\hline DCSI quartile & & & & & & & $* *$ \\
\hline 0 to 0 & 846 & 31.9 & 305 & 32.2 & 541 & 31.7 & \\
\hline 1 to 1 & 476 & 17.9 & 195 & 20.6 & 281 & 16.5 & \\
\hline 2 to 3 & 811 & 30.6 & 297 & 31.3 & 514 & 30.2 & \\
\hline 4 to 13 & 519 & 19.6 & 151 & 15.9 & 368 & 21.6 & \\
\hline
\end{tabular}

Notes: based on the data of 2,652 elderly men aged 66 years and older diagnosed with prostate cancer between 2008 and 2009 using a surveillance, epidemiology, and end-results- (SEER-) Medicare linked Part D data. Significant group differences by metformin use are based on chi-square tests. \% represented in the column is row percentages.

DCSI: diabetes complication severity index; PCP: primary care physician; PSA: prostate specific antigen; Sig.: level of significance. ${ }^{* * *} p<0.001 ;{ }^{* *} 0.001 \leq$ $p<0.01 ;{ }^{*} 0.01 \leq p<0.05$.

enrolled in Parts A, B, and D as shown in the Appendix. These two sets of population shared similar characteristics.

\subsection{Key Dependent Variable}

2.4.1. Cancer Stage at Diagnosis. The American Joint Committee on Cancer (AJCC) Tumor-Node-Metastases (TNM) classification was used to identify the stage of prostate cancer from the PEDSF file. Based on the AJCC-TNM systems, men were classified as having localized cancer stage if they had $\mathrm{T} 1$ or T2 clinical stage with no regional lymph node (NXN0) involvement and absence of any distant metastasis (M0) [21]. Men were classified as having advanced cancer if they were diagnosed with T3 or T4 clinical stage with or without regional lymph node (N1) or distant metastasis (M1).

\subsection{Key Independent Variable}

2.5.1. Metformin Use. Metformin use was identified using the Medicare Part D files. Metformin prescriptions were identified using the national drug codes (NDCs). Men with at least one prescription for metformin during the baseline period were considered as metformin users and men without any prescriptions for metformin were considered as nonusers.

\subsection{Other Independent Variables}

2.6.1. Conceptual Framework. We utilized the Anderson Healthcare Behavior and Utilizations Model (ABM) model [22] to classify the potential independent factors associated with advanced prostate cancer.

Predisposing Characteristics. Predisposing characteristics consisted of age at diagnosis, race, and marital status. These were identified from the PEDSF. Age at the time of diagnosis was categorized into two groups (66 to 74 years, $\geq 75$ years). Race/ethnicity was categorized into four groups: White, African American, Latino, and others. Marital status was categorized into four groups: married, divorced/separated, unmarried, and others.
Enabling Characteristics. Enabling characteristics were as follows: education, income, prostate specific antigen test, and visits to primary care physicians. Median income and median education at the census tract of residence were derived from the PEDSF. Income and education were measured by quartiles. The receipt of PSA test was identified using the following HCPCS codes: 84152, 84153, 84154, and G0103 using the Medicare carrier files during the baseline period. The presence or absence of primary care visits during the baseline was identified using the provider specialty codes [23].

Need Characteristics. The presence of T2DM was identified using at least one inpatient visit or two or more physician visits with a primary or a secondary diagnosis codes for T2DM (ICD-9-CM codes: 250.x0 or 250.x2) during the baseline period. The severity of diabetes, oral antidiabetic medication use, insulin use, statins use, and corticosteroid use were considered as need factors. The Diabetes Complications Severity Index (DCSI) was calculated using the modified algorithm by Chang et al. [24]. The DSCI is based on seven categories: retinopathy, nephropathy, neuropathy, cerebrovascular, cardiovascular, peripheral vascular disease, and metabolic conditions. Based on the severity of particular types of complications, a score of 1 or 2 was assigned to each of the seven categories, with a total DCSI score ranging from 0 to 13 . The DCSI scores were grouped into quartiles. Details of the ICD-9-CM codes and the scoring algorithm are provided in the Appendix. The use of oral antidiabetic medications, insulin, statins, and corticosteroids was identified using the NDCs recorded in the Medicare Part D files during the baseline period.

External Environment Characteristics. The SEER data has 18 registries/regions which were categorized into four regions: (1) Northeast, with two registries of Connecticut and New Jersey; (2) South, with five registries of Kentucky, Louisiana, Atlanta, Rural Georgia, and Greater Georgia; (3) Northcentral, with two registries of Detroit and Iowa; and (4) West, with Hawaii, New Mexico, Seattle-Puget Sound, Utah, San Francisco-Oakland, San Jose-Monterey, Los Angeles, Greater California, Arizona, Alaska, and Cherokee Nation. 
TABLE 2: Adjusted odds ratios and 95\% confidence intervals from logistic regressions on metformin use among elderly Medicare beneficiaries with diabetes incident prostate cancer surveillance, epidemiology, and end-results- (SEER-) Medicare linked data, $2007-2010$.

\begin{tabular}{|c|c|c|c|}
\hline & AOR & $95 \% \mathrm{CI}$ & Sig. \\
\hline \multicolumn{4}{|c|}{ Predisposing characteristics } \\
\hline \multicolumn{4}{|l|}{ Age at diagnosis } \\
\hline $66-74$ years & 1.31 & {$[1.10,1.56]$} & $* *$ \\
\hline 75 years or above & Ref. & & \\
\hline \multicolumn{4}{|l|}{ Race/ethnicity } \\
\hline Whites & Ref. & & \\
\hline African American & 0.91 & {$[0.69,1.21]$} & \\
\hline Latino & 1.62 & {$[1.12,2.34]$} & $* *$ \\
\hline Others & 0.80 & {$[0.59,1.10]$} & \\
\hline \multicolumn{4}{|l|}{ Marital status } \\
\hline Married & Ref. & & \\
\hline Unmarried & 0.81 & {$[0.59,1.11]$} & \\
\hline Divorced/separated & 0.94 & {$[0.74,1.20]$} & \\
\hline Others & 0.83 & {$[0.66,1.04]$} & \\
\hline \multicolumn{4}{|l|}{ Enabling characteristics } \\
\hline \multicolumn{4}{|c|}{ Quartile of median census of 2000 income } \\
\hline$\$ 7-\$ 34,522$ & 1.72 & {$[1.20,2.46]$} & $* *$ \\
\hline$\$ 34,523-46,224$ & 1.34 & {$[0.97,1.84]$} & \\
\hline$\$ 46,229-62,764$ & 1.18 & {$[0.89,1.56]$} & \\
\hline$\$ 62,767-200,008$ & Ref. & & \\
\hline \multicolumn{4}{|c|}{ Quartile of median census of 2000 education } \\
\hline $0-8.52$ & 1.24 & {$[0.87,1.77]$} & \\
\hline $8.53-15.16$ & 1.21 & {$[0.90,1.62]$} & \\
\hline $15.17-26.09$ & 1.18 & {$[0.92,1.51]$} & \\
\hline $26.1-100$ & Ref & & \\
\hline \multicolumn{4}{|l|}{ Visit to a $P C P$} \\
\hline Yes & 1.12 & {$[0.92,1.35]$} & \\
\hline No & Ref. & & \\
\hline \multicolumn{4}{|l|}{ Statin use } \\
\hline Yes & 1.94 & {$[1.63,2.31]$} & $* * *$ \\
\hline No & Ref & & \\
\hline \multicolumn{4}{|l|}{ Insulin } \\
\hline Yes & 2.95 & {$[1.94,4.49]$} & $* * *$ \\
\hline No & Ref. & & \\
\hline \multicolumn{4}{|l|}{ Need factors } \\
\hline \multicolumn{4}{|l|}{ DCSI quartile } \\
\hline 0 to 1 & Ref. & & \\
\hline 2 to 2 & 1.13 & {$[0.89,1.44]$} & \\
\hline 3 to 3 & 0.98 & {$[0.79,1.21]$} & \\
\hline 4 to 13 & 0.65 & {$[0.51,0.84]$} & $* * *$ \\
\hline \multicolumn{4}{|c|}{ External environment characteristics } \\
\hline \multicolumn{4}{|l|}{ SEER-regions } \\
\hline Northeast & Ref. & & \\
\hline South & 1.40 & {$[1.06,1.85]$} & * \\
\hline North-central & 1.07 & {$[0.78,1.47]$} & \\
\hline West & 1.37 & {$[1.08,1.75]$} & * \\
\hline
\end{tabular}

Notes: based on the data of 2,652 elderly men aged 66 years and older diagnosed with prostate cancer between 2008 and 2009 using a surveillance, epidemiology, and end-results- (SEER-) Medicare linked Part D data. Significant group differences are based on log-likelihood test for metformin use.

DCSI: diabetes complication severity index; PCP: primary care physician; Ref.: reference group; Sig.: level of significance; ${ }^{* * *} p<0.001 ;{ }^{* *} 0.001 \leq p<0.01$; ${ }^{*} 0.01 \leq p<0.05$. 
TABLE 3: (a) Number and IPTW-adjusted percentage of men with localized versus advanced stage of prostate cancer by metformin use among elderly Medicare beneficiaries with diabetes and incident prostate cancer surveillance, epidemiology, and end-results- (SEER) Medicare linked database, 2007-2010. (b) Unadjusted and adjusted odds ratios and 95\% confidence intervals from (IPTW) logistic regressions for advanced stage at diagnosis of cancer.

(a)

\begin{tabular}{|c|c|c|c|c|c|}
\hline \multirow[b]{2}{*}{ Overall } & \multicolumn{2}{|c|}{ Localized } & \multicolumn{2}{|r|}{ Advance } & \multirow{2}{*}{ Sig } \\
\hline & $N$ & Weighted\% & $N$ & Weighted\% & \\
\hline & 2,493 & 93.7 & 159 & 6.3 & \\
\hline Metformin use & & & & & * \\
\hline Yes & 902 & 95.3 & 46 & 4.7 & \\
\hline No & 1,591 & 93.3 & 113 & 6.7 & \\
\hline
\end{tabular}

(b)

\begin{tabular}{|c|c|c|c|}
\hline \multicolumn{4}{|c|}{ Advanced stage at diagnosis IPTW } \\
\hline \multicolumn{4}{|c|}{ Unadjusted odds ratios and 95\% confidence intervals } \\
\hline & OR & 95\% CI & Sig. \\
\hline \multicolumn{4}{|c|}{ Metformin } \\
\hline Yes & 0.69 & {$[0.49,0.95]$} & * \\
\hline No & Ref. & & \\
\hline \multicolumn{4}{|c|}{ Adjusted odds ratios and $95 \%$ confidence intervals } \\
\hline & $\mathrm{AOR}$ & $95 \% \mathrm{CI}$ & Sig. \\
\hline \multicolumn{4}{|c|}{ Metformin } \\
\hline Yes & 0.68 & {$[0.48,0.97]$} & * \\
\hline No & Ref. & & \\
\hline
\end{tabular}

Notes: based on the data of 2,652 elderly men aged 66 years and older diagnosed with prostate cancer between 2008 and 2009 using a surveillance, epidemiology, and end-results- (SEER-) Medicare linked data. \% is weighted percentage for IPTW. Significant differences are based on the log-likelihood test using a logistic regression with IPTW weights. Adjusted model controlled for predisposing, enabling, need, and external environment related factors.

IPTW: inverse probabilities treatment weights; PSA: prostate specific antigen level; PCP: primary care physician visit; Sig.: level of significance; ${ }^{* * *} p<$ $0.001 ;{ }^{* *} 0.001 \leq p<0.01 ;{ }^{*} 0.01 \leq p<0.05$.

The Area Healthcare Resource Use Files (AHRF) were linked to identify the number of radiation oncology units, and urology units at the county-level with cancer cases and quartiles of total radiation oncology and urology units were created for each case [25].

2.7. Statistical Analyses. Significant group differences in the study population characteristics by metformin use were examined with chi-square tests. A binary logistic regression was used to determine the associations between predisposing, enabling, need, and external environment characteristics and metformin use. C-statistics and area under the curve were used to assess the model fit. The logistic regression was used to derive inverse probability treatment weights (IPTWs) and these standardized IPTWs were used to control for the observed selection bias in regressions on the cancer stage.

Significant unadjusted associations between metformin use and cancer stage at diagnosis were examined with chi-square tests. The IPTW-adjusted multivariable logistic regressions were used to analyze the relationship between metformin use and cancer stage at diagnosis. As the odds ratios and relative risk are approximately similar for the events with low prevalence, such as advanced prostate cancer $(\leq 10 \%)$ [26], these terms risk ratio or odds ratio of advanced prostate cancer were used interchangeably. All statistical analyses were carried out using Statistical Analysis System (SAS) version 9.4 (SAS Institute Inc., Cary, NC).

\section{Results}

3.1. Description of the Study Cohort. The study cohort consisted of 2,652 elderly men with preexisting T2DM and incident prostate cancer between 2008 and 2009. Table 1 represents the characteristics of the study cohort. An overwhelming majority of men were Whites (92.3); 59.7\% were diagnosed with prostate cancer between the ages of 66 and 74 years; $58.6 \%$ were married; and $46.2 \%$ resided in the Western region of the US. Nearly three-quarters (70.0\%) of study cohort had a primary care visit. An overwhelming majority (91.1\%) had PSA test during the baseline period.

3.2. Description of the Study Cohort by Metformin Use. Table 1 also summarizes the characteristics of the study cohort by metformin use. Overall, $35.6 \%$ of the study cohort had at least one prescription of metformin during the baseline period. Significant differences in predisposing, enabling, need, and external environment factors by metformin use were observed.

Table 2 describes the adjusted odds ratios (AOR) and 95\% $\mathrm{CI}$ for the metformin use among elderly men with diabetes and prostate cancer. Elderly men aged 66 to 74 years as compared to those aged 75 years and older (AOR: 1.31, 95\% CI: 1.10, 1.56), Latinos as compared to Whites (AOR: 1.62, 95\% CI: 1.12, 2.34), those who received insulin as compared to no insulin (AOR: 2.95, 95\% CI: 1.94, 4.49), and those who received statins as compared to no statins (AOR: 1.94, 95\% CI: $1.63,2.31$ ) were more likely to receive metformin. Whereas, elderly men with a severe DCSI score (4 to 13) were more likely to receive metformin as compared to those with zero or one DCSCI score (AOR: $0.65,95 \%$ CI: $0.51,0.84$ ).

3.3. Metformin and Cancer Stage at Diagnosis. Table 3 describes the relationship between metformin use and cancer stage at diagnosis among elderly men with prostate cancer and diabetes. Overall, $93.7 \%$ of the study population was diagnosed with localized prostate cancer; $6.3 \%$ was diagnosed with advanced prostate cancer. A significantly lower percentage of metformin users were diagnosed with advanced prostate cancer as compared to nonusers $(4.7 \%$ versus $6.7 \%$, $p<0.03)$.

Table 3 also reports unadjusted odds ratios (OR) and adjusted ORs (AOR) from IPTW logistic regressions for the advanced prostate cancer among elderly men with T2DM and prostate cancer. In unadjusted logistic regression, we observed the association between metformin and risk of advanced prostate cancer at diagnosis (OR: 0.69, 95\% CI: $0.49,0.95)$. After adjusting for predisposing, enabling, need, 
TABLE 4: Codes and algorithms to identify diabetes complication severity index (DCSI) developed by Young et al. and modified by Chang et al.

\begin{tabular}{|c|c|c|}
\hline Complications & ICD-9-CM code & DCSI score \\
\hline \multicolumn{3}{|l|}{ Retinopathy } \\
\hline Diabetic ophthalmologic disease & $250.5 x$ & 1 \\
\hline Background retinopathy & 362.01 & 1 \\
\hline Other retinopathies & 362.1 & 1 \\
\hline Retinal edema & 362.83 & 1 \\
\hline CSME & 362.53 & 1 \\
\hline Other retinal disorders & $362.81,362.82$ & 1 \\
\hline Proliferative retinopathy & 362.02 & 2 \\
\hline Retinal detachment & 361.xx & 2 \\
\hline Blindness & 369.xx.00-0.99 & 2 \\
\hline Vitreous hemorrhage & 379.23 & 2 \\
\hline \multicolumn{3}{|l|}{ Nephropathy } \\
\hline Diabetic nephropathy & 250.4 & 1 \\
\hline Acute glomerulonephritis & 580 & 1 \\
\hline Nephrotic syndrome & 581 & 1 \\
\hline Hypertension, nephrosis & 581.81 & 1 \\
\hline Chronic glomerulonephritis & 582 & 1 \\
\hline Nephritis/nephropathy & 583 & 1 \\
\hline Chronic renal failure & 585 & \\
\hline Renal failure NOS & 586 & \\
\hline Renal insufficiency & 593.9 & \\
\hline \multicolumn{3}{|l|}{ Neuropathy } \\
\hline Diabetic nephropathy & $356.9,250.6$ & 1 \\
\hline Amyotrophy & 358.1 & 1 \\
\hline Cranial nerve palsy & $951.0,951.1,951.3$ & 1 \\
\hline Mononeuropathy & $354.0-355.9$ & 1 \\
\hline Charcot's arthropathy & 713.5 & 1 \\
\hline Polyneuropathy & 357.2 & 1 \\
\hline \multicolumn{3}{|l|}{ Cerebrovascular } \\
\hline TIA & 435 & 1 \\
\hline Stroke & $431,433,434,436$ & 2 \\
\hline \multicolumn{3}{|l|}{ Cardiovascular } \\
\hline Atherosclerosis & $440 . x x$ & 1 \\
\hline Other IHD & 411 & 1 \\
\hline Angina pectoris & 413 & 1 \\
\hline Other chronic IHD & 414 & 1 \\
\hline Myocardial infarction & 410 & 2 \\
\hline Ventricular fibrillation, arrest & $427.1,427.3$ & 2 \\
\hline Atrial fibrillation, arrest & $427.4,427.5$ & 2 \\
\hline Other ASCVD & 429.2 & 1 \\
\hline Old myocardial infarction & 412 & 2 \\
\hline Heart failure & 428 & 2 \\
\hline Atherosclerosis, severe & $440.23,440.24$ & 2 \\
\hline Aortic aneurysm/dissection & 441 & 2 \\
\hline \multicolumn{3}{|l|}{ Peripheral vascular disease } \\
\hline Diabetic PVD & 250.7 & 1 \\
\hline Other aneurysms, LE & 442.3 & 1 \\
\hline PVD & $443.81,443.9$ & 1 \\
\hline
\end{tabular}


TABLE 4: Continued.

\begin{tabular}{lcc}
\hline Complications & ICD-9-CM code & DCSI score \\
\hline Foot wound + complication & 892.1 & 1 \\
Claudication, intermittent & 443.9 & 1 \\
Embolism/thrombosis (LE) & 444.22 & 2 \\
Gangrene & 785.4 & 2 \\
Gas gangrene & 0.4 & 2 \\
Ulcer of lower limbs & 707.1 & 2 \\
\hline Metabolic & & 250.1 \\
Ketoacidosis & 250.2 & 2 \\
Hyperosmolar & 250.3 & 1 \\
Other comas & & \\
\hline
\end{tabular}

Note: the table is adapted from the previous algorithm defined by Young et al. and modified by Change et al. to identify the severity of diabetes using claims database. Severity index was based on a scale ranging from 0 to 2 for each complication as follows: $0=$ no abnormality, $1=$ some abnormality, and $2=$ severe abnormality.

ASCVD, atherosclerotic cardiovascular disease; CSME, cystoid macular edema/degeneration; DCSI, diabetes complications severity index; IHD, ischemic heart disease; ICD-9-CM, international classification of diseases, ninth revision, clinical modification; LE, lower extremity; NOS, not otherwise specified; PVD, peripheral vascular disease; TIA, transient ischemic attack.

and external environment factors among elderly men with prostate cancer and diabetes, metformin use was significantly associated with a reduction in the risk of advanced prostate cancer (AOR: 0.68, 95\% CI: 0.48, 0.97).

\section{Discussion}

The current study is the first largest population-based study to examine whether the risk of advanced prostate cancer diagnosis is reduced with metformin use among elderly men with preexisting T2DM and incident prostate cancer in the US. Characteristics of metformin users were consistent with literature. We observed that a higher percentage of men living in the Western region of the united states were prescribed metformin as compared to those living in other regions. Census-track level income was also associated with metformin use. These study findings are consistent with one published study on geographical disparities in antidiabetes medications. Regional disparities in metformin use can reflect practice patterns in regions [26].

We found that the risk for advanced stage cancer diagnosis is reduced with metformin use among elderly men with T2DM and incident prostate cancer after controlling for the observed section bias between metformin users and nonusers and other independent variables. The current study addressed the limitations of the single population-based examination of metformin use and the cancer stage by incorporating a validated diabetes severity complications index and controlling for the observed selection bias. The current study findings are consistent with the preclinical evidence on the role of metformin in prevention of advanced prostate cancer [10, 27]. Further, it should be noted that neither oral antidiabetic medications (sulfonylurea and thiazolidinedione) nor insulin use was associated with advanced prostate cancer (data not shown in tabular format). Therefore, it is plausible that metformin may act via insulin-independent pathways to reduce the risk of advanced prostate cancer. If the findings of the current study are confirmed by other populationbased studies, randomized clinical trials can be conducted to establish the causal link between metformin use and risk of advanced prostate cancer diagnosis.

It should be noted that the current study controlled for the observed selection bias because metformin users and nonusers were significantly different with respect to their predisposing, enabling, need, and external environment characteristics. Without adjustments for the observed selection bias, there was not a statistically significant difference in the cancer stage between metformin users and nonusers. Therefore, accounting for the observed selection bias is important in establishing an association between metformin use and the reduction in the risk of advanced prostate cancer diagnosis.

The current study has a number of strengths. The large cohort size and high-quality data on the clinical and pathological features of cancer at the time of diagnosis enabled us to examine not only the association between metformin use and the cancer stage diagnosis but also the initial choice of cancer treatment [18]. Furthermore, the inclusion of variable on severity of diabetes using a validated method enabled controlling and relating the effect of severity of disease on the risk of advanced prostate cancer at diagnosis and receipt of initial cancer treatment.

The current study has some limitations as well. The prescription claims for metformin and other drugs were used. Filling the prescriptions cannot be equated to the actual use of these drugs. We made an attempt to overcome this issue to some extent via measuring the one-year adherence to medication, and a lower proportion of those with adherence to metformin and nonadherent metformin had advanced prostate cancer as compared to nonusers. Due to lack of sufficient sample size, we did not present the results in tabular format. However, the direction of our findings is consistent with the previous studies suggesting no difference in the ever metformin users and adherent metformin users on grade of prostate cancer [15]. Secondly, we focused on elderly men with prostate cancer because two-thirds of prostate cancers cases are diagnosed among the elderly men aged 65 years and 
TABLE 5: Baseline characteristics of elderly men with prostate cancer with T2DM overall and enrolled in Part D program SEER-Medicare linked database, 2008-2009.

\begin{tabular}{|c|c|c|}
\hline \multirow{2}{*}{ All } & DM Part D $(N=2,652)$ & DM overall $(N=7,424)$ \\
\hline & $\%$ & $\%$ \\
\hline \multicolumn{3}{|l|}{ Age at diagnosis, in years } \\
\hline $66-74$ & 59.5 & 59.4 \\
\hline $75+$ & 40.5 & 40.6 \\
\hline \multicolumn{3}{|l|}{ Race/ethnicity } \\
\hline Whites & 72.2 & 75.3 \\
\hline African American & 12.2 & 14.5 \\
\hline Hispanic/Latino & 5.7 & 3.0 \\
\hline Others & 10.0 & 7.1 \\
\hline \multicolumn{3}{|l|}{ Marital status } \\
\hline Unmarried & 8.4 & 6.6 \\
\hline Married & 58.4 & 63.2 \\
\hline Divorced/separated & 14.8 & 14.0 \\
\hline Others & 18.4 & 16.2 \\
\hline \multicolumn{3}{|c|}{ Quartile of median census of 2000 income } \\
\hline$\$ 7-\$ 34,522$ & 25.0 & 24.9 \\
\hline$\$ 34,523-46,224$ & 25.0 & 25.1 \\
\hline$\$ 46,229-62,764$ & 25.0 & 25.0 \\
\hline$\$ 62,767-200,008$ & 24.9 & 25.0 \\
\hline \multicolumn{3}{|c|}{ Quartile of median census of 2000 education } \\
\hline $0-8.52$ & 25.1 & 25.0 \\
\hline $8.53-15.16$ & 24.7 & 25.0 \\
\hline $15.17-26.09$ & 25.0 & 25.1 \\
\hline $26.1-100$ & 25.2 & 25.0 \\
\hline \multicolumn{3}{|l|}{ Visit to a $P C P$} \\
\hline Yes & 70.1 & 65.0 \\
\hline No & 29.9 & 35.0 \\
\hline \multicolumn{3}{|c|}{ PSA screening in past year } \\
\hline Yes & 91.1 & 88.4 \\
\hline No & 8.9 & 11.6 \\
\hline \multicolumn{3}{|l|}{ SEER-regions } \\
\hline Northeast & 18.4 & 19.3 \\
\hline South & 21.5 & 24.9 \\
\hline North-central & 13.2 & 11.8 \\
\hline West & 46.9 & 44.0 \\
\hline \multicolumn{3}{|c|}{ Quartile of radiation oncology } \\
\hline 0 to 1 & 26.1 & 26.1 \\
\hline 2 to 6 & 24.7 & 24.7 \\
\hline 7 to 22 & 25.3 & 25.3 \\
\hline 23 to 147 & 23.9 & 23.9 \\
\hline \multicolumn{3}{|c|}{ Quartile of urology centers } \\
\hline 0 to 3 & 24.5 & 24.5 \\
\hline 4 to 16 & 24.7 & 24.7 \\
\hline 17 to 44 & 26.2 & 26.2 \\
\hline 45 to 343 & 24.5 & 24.5 \\
\hline \multicolumn{3}{|l|}{ Year of diagnosis } \\
\hline 2008 & 50.9 & 51.6 \\
\hline 2009 & 49.1 & 48.4 \\
\hline
\end{tabular}


older [28]; however, the study population consisted of elderly men with T2DM residing in SEER-regions and enrolled in fee-for-services Medicare Parts A, B, and D plans; therefore, one cannot generalize the study finding to younger men or all Medicare beneficiaries with incident prostate cancer in the US. Information on many important prognostic factors such as body-mass index and smoking could not be adjusted; these factors may be associated with an increased risk of advanced prostate cancer diagnosis. Duration of metformin use could not be adjusted due to data limitations. Future studies need to examine whether a greater duration of metformin use is associated with a decrease in the risk of advanced prostate cancer at diagnosis. As the study population was restricted to elderly men with T2DM, the current study findings cannot be generalized to men without T2DM and incident prostate cancer.

\section{Conclusions}

Metformin use was associated with a statistically significant reduction in the risk advanced prostate cancer diagnosis among elderly men with T2DM and incident prostate cancer. The current study findings highlight the need for additional studies in this area. Other population-based studies need to be conducted to confirm the study findings. If confirmed, randomized controlled trials can be carried out to examine the causal link between metformin use and the risk of advanced prostate cancer diagnosis.

\section{Appendix}

See Tables 4 and 5.

\section{Competing Interests}

The authors have no competing interests to declare.

\section{Authors' Contributions}

Amit D. Raval and Usha Sambamoorthi wrote the paper and researched data. Suresh Madhavan, Malcolm D. Mattes, Wenhui Wei, and Xiaoyun Pan reviewed/edited the paper.

\section{Acknowledgments}

This project was supported by Agency for Health Research and Quality (AHRQ) Grant no. R24HS018622-03 and National Institute of General Medicine Sciences (Grant U54GM104942). The content is solely the responsibility of the authors and does not necessarily represent the official views of AHRQ and NIH.

\section{References}

[1] E. Giovannucci, D. M. Harlan, M. C. Archer et al., "Diabetes and cancer: a consensus report," Diabetes Care, vol. 33, no. 7, pp. 1674-1685, 2010.
[2] F. Abdollah, A. Briganti, N. Suardi et al., "Does diabetes mellitus increase the risk of high-grade prostate cancer in patients undergoing radical prostatectomy?" Prostate Cancer and Prostatic Diseases, vol. 14, no. 1, pp. 74-78, 2011.

[3] D. Bansal, A. Bhansali, G. Kapil, K. Undela, and P. Tiwari, "Type 2 diabetes and risk of prostate cancer: a meta-analysis of observational studies," Prostate Cancer and Prostatic Diseases, vol. 16, no. 2, supplement 1, pp. 151-158, 2013.

[4] J. Kang, M.-H. Chen, Y. Zhang et al., "Type of diabetes mellitus and the odds of Gleason score 8 to 10 prostate cancer," International Journal of Radiation Oncology Biology Physics, vol. 82, no. 3, pp. e463-e467, 2012.

[5] A. M. Ribeiro, S. Pereira, S. Andrade et al., "Insulin prevents leptin inhibition of RM1 prostate cancer cell growth," Pathology and Oncology Research, vol. 18, no. 2, pp. 499-507, 2012.

[6] A. A. Lubik, J. H. Gunter, S. C. Hendy et al., "Insulin increases de novo steroidogenesis in prostate cancer cells," Cancer Research, vol. 71, no. 17, pp. 5754-5764, 2011.

[7] H. Xu, H.-W. Jiang, G.-X. Ding et al., "Diabetes mellitus and prostate cancer risk of different grade or stage: a systematic review and meta-analysis," Diabetes Research and Clinical Practice, vol. 99, no. 3, pp. 241-249, 2013.

[8] M. Khera, D. Crawford, A. Morales, A. Salonia, and A. Morgentaler, "A new era of testosterone and prostate cancer: from physiology to clinical implications," European Urology, vol. 65, no. 1, pp. 115-123, 2014.

[9] N. Ashokkumar, L. Pari, and C. A. Rao, "Effect of N-benzoyl-Dphenylalanine and metformin on insulin receptors in neonatal streptozotocin-induced diabetic rats: studies on insulin binding to erythrocytes," Archives of Physiology and Biochemistry, vol. 112, no. 3, pp. 174-181, 2006.

[10] S. M. Hadad, S. Fleming, and A. M. Thompson, "Targeting AMPK: a new therapeutic opportunity in breast cancer," Critical Reviews in Oncology/Hematology, vol. 67, no. 1, pp. 1-7, 2008.

[11] X. Huang, S. Wullschleger, N. Shpiro et al., "Important role of the LKB1-AMPK pathway in suppressing tumorigenesis in PTEN-deficient mice," Biochemical Journal, vol. 412, no. 2, pp. 211-221, 2008.

[12] R. L. Bitting and A. J. Armstrong, "Targeting the $\mathrm{PI} 3 \mathrm{~K} / \mathrm{Akt} / \mathrm{mTOR}$ pathway in castration-resistant prostate cancer," Endocrine-Related Cancer, vol. 20, no. 3, pp. R83-R99, 2013.

[13] D. Deng, Y. Yang, X. Tang et al., "Association between metformin therapy and incidence, recurrence and mortality of prostate cancer: evidence from a meta-analysis," Diabetes/Metabolism Research and Reviews, vol. 31, no. 6, pp. 595602, 2015.

[14] I. B. Sahra, K. Laurent, A. Loubat et al., "The antidiabetic drug metformin exerts an antitumoral effect in vitro and in vivo through a decrease of cyclin D1 level," Oncogene, vol. 27, no. 25, pp. 3576-3586, 2008.

[15] D. Margel, D. Urbach, L. L. Lipscombe et al., "Association between metformin use and risk of prostate cancer and its grade," Journal of the National Cancer Institute, vol. 105, no. 15, pp. 1123-1131, 2013.

[16] H. Müller, E. Raum, D. Rothenbacher, C. Stegmaier, and H. Brenner, "Association of diabetes and body mass index with levels of prostate-specific antigen: implications for correction of prostate-specific antigen cutoff values?" Cancer Epidemiology Biomarkers \& Prevention, vol. 18, no. 5, pp. 1350-1356, 2009.

[17] Surveillance, Epidemiology, and End Results (SEER) Program, SEER ${ }^{\star}$ Stat Database: Incidence-SEER 18 Regs Public Use, Nov. 
2012 Sub (2000-2010)_Linked to County Attributes_Total US, 1969-2011 Counties, National Cancer Institute, Division of Cancer Control and Population Sciences, Surveillance Research Program, Cancer Statistics Branch, Bethesda, Md, USA, 2013.

[18] J. L. Warren, C. N. Klabunde, D. Schrag, P. B. Bach, and G. F. Riley, "Overview of the SEER-Medicare data: content, research applications, and generalizability to the United States elderly population," Medical Care, vol. 40, no. 8, supplement, pp. 3-18, 2002.

[19] "SEER-Medicare: How the SEER \& Medicare data are linked?" 2013, http://appliedresearch.cancer.gov/seermedicare/aboutdata/.

[20] "Number of SEER-Medicare Patients with Part D Enrollment by Calendar Year Diagnosis Years 1986-2007," 2013, http:// appliedresearch.cancer.gov/seermedicare/aboutdata/enrollees .html.

[21] I. Thompson, J. B. Thrasher, G. Aus et al., "Guideline for the management of clinically localized prostate cancer: 2007 update," The Journal of Urology, vol. 177, no. 6, pp. 2106-2131, 2007.

[22] R. M. Andersen, "Revisiting the behavioral model and access to medical care: does it matter?" Journal of Health and Social Behavior, vol. 36, no. 1, pp. 1-10, 1995.

[23] J. B. Yu, P. R. Soulos, J. Herrin et al., "Proton versus intensitymodulated radiotherapy for prostate cancer: patterns of care and early toxicity," Journal of the National Cancer Institute, vol. 105, no. 1, pp. 25-32, 2013.

[24] H. Y. Chang, J. P. Weiner, T. M. Richards, S. N. Bleich, and J. B. Segal, "Validating the adapted Diabetes Complications Severity Index in claims data," The American Journal of Managed Care, vol. 18, no. 11, pp. 721-726, 2012.

[25] Area Resource File: Overview, 2014, http://ahrf.hrsa.gov/overview.htm.

[26] M. R. Sargen, O. J. Hoffstad, D. J. Wiebe, and D. J. Margolis, "Geographic variation in pharmacotherapy decisions for U.S. medicare enrollees with diabetes," Journal of Diabetes and its Complications, vol. 26, no. 4, pp. 301-307, 2012.

[27] L. C. Trantham, M. E. Nielsen, L. R. Mobley, S. B. Wheeler, W. R. Carpenter, and A. K. Biddle, "Use of prostate-specific antigen testing as a disease surveillance tool following radical prostatectomy," Cancer, vol. 119, no. 19, pp. 3523-3530, 2013.

[28] R. L. Siegel, K. D. Miller, and A. Jemal, "Cancer statistics, 2015," CA: A Cancer Journal for Clinicians, vol. 65, no. 1, pp. 5-29, 2015. 


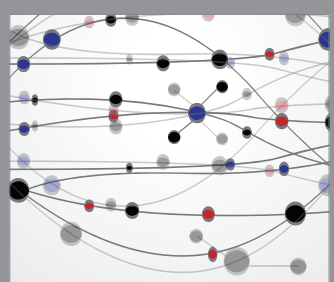

The Scientific World Journal
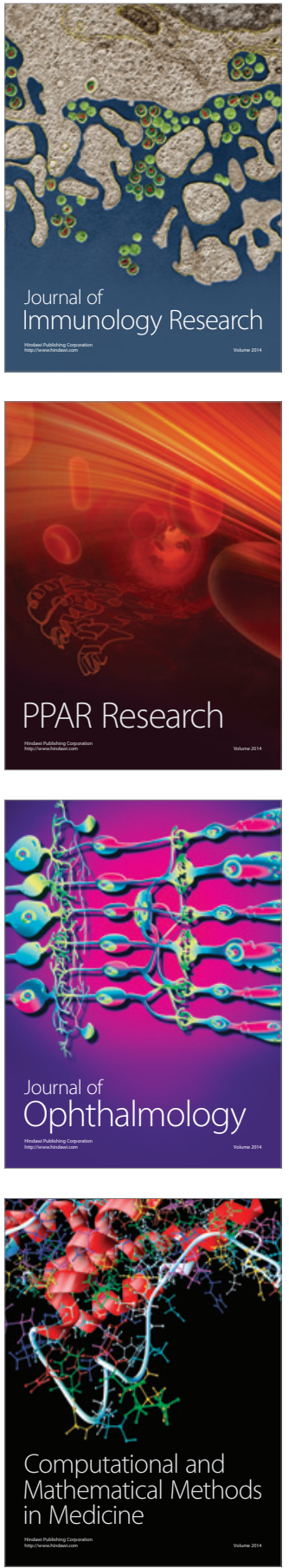

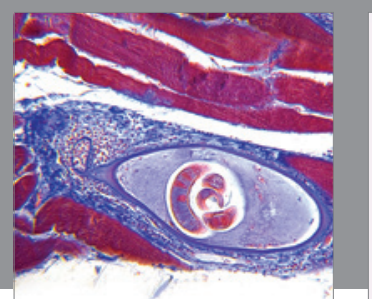

Gastroenterology Research and Practice

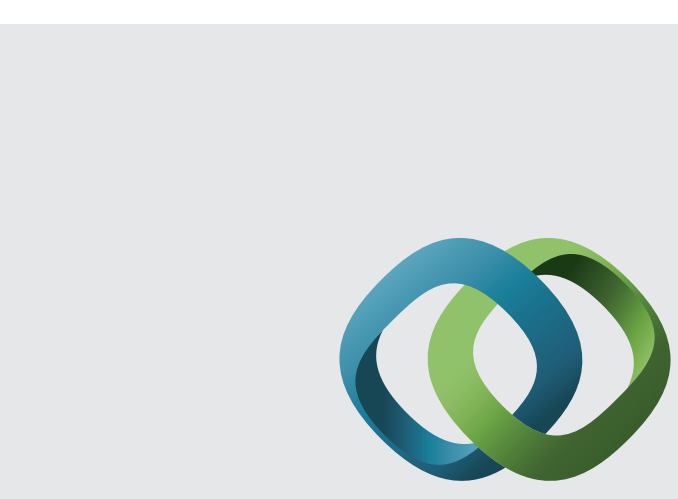

\section{Hindawi}

Submit your manuscripts at

http://www.hindawi.com
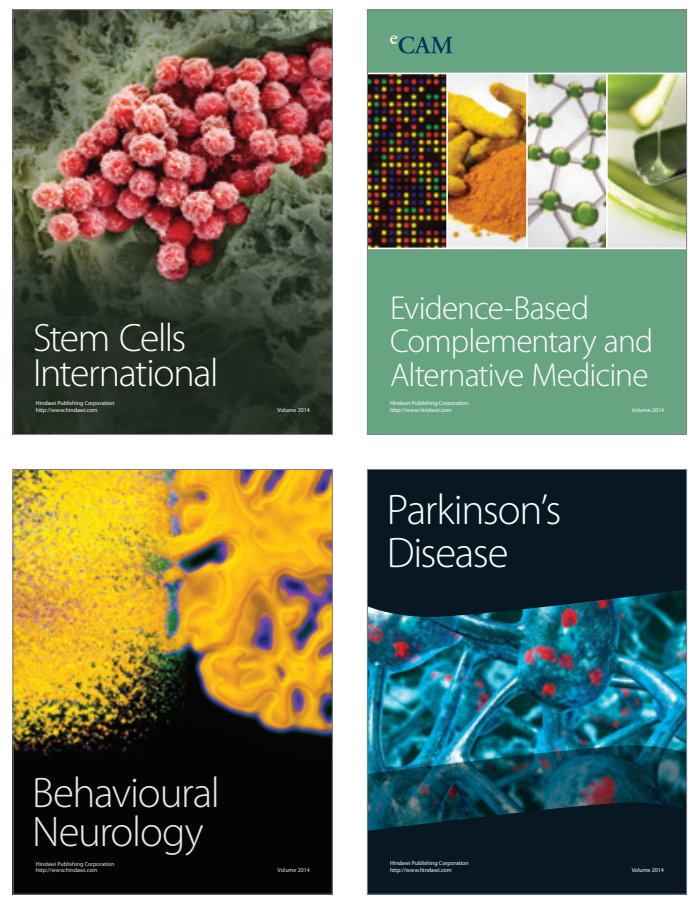
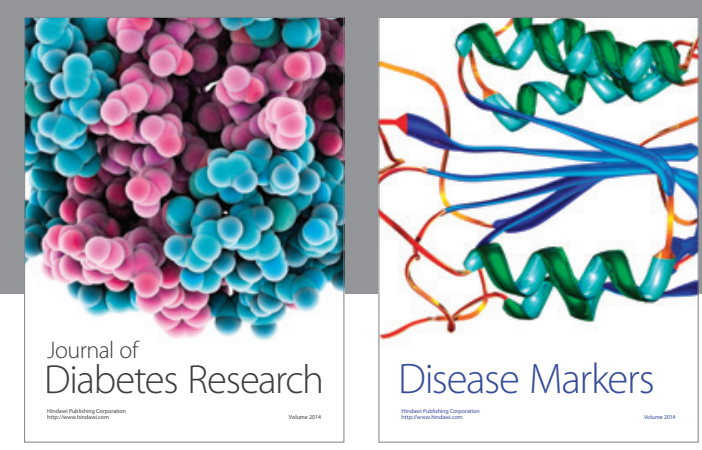

Disease Markers
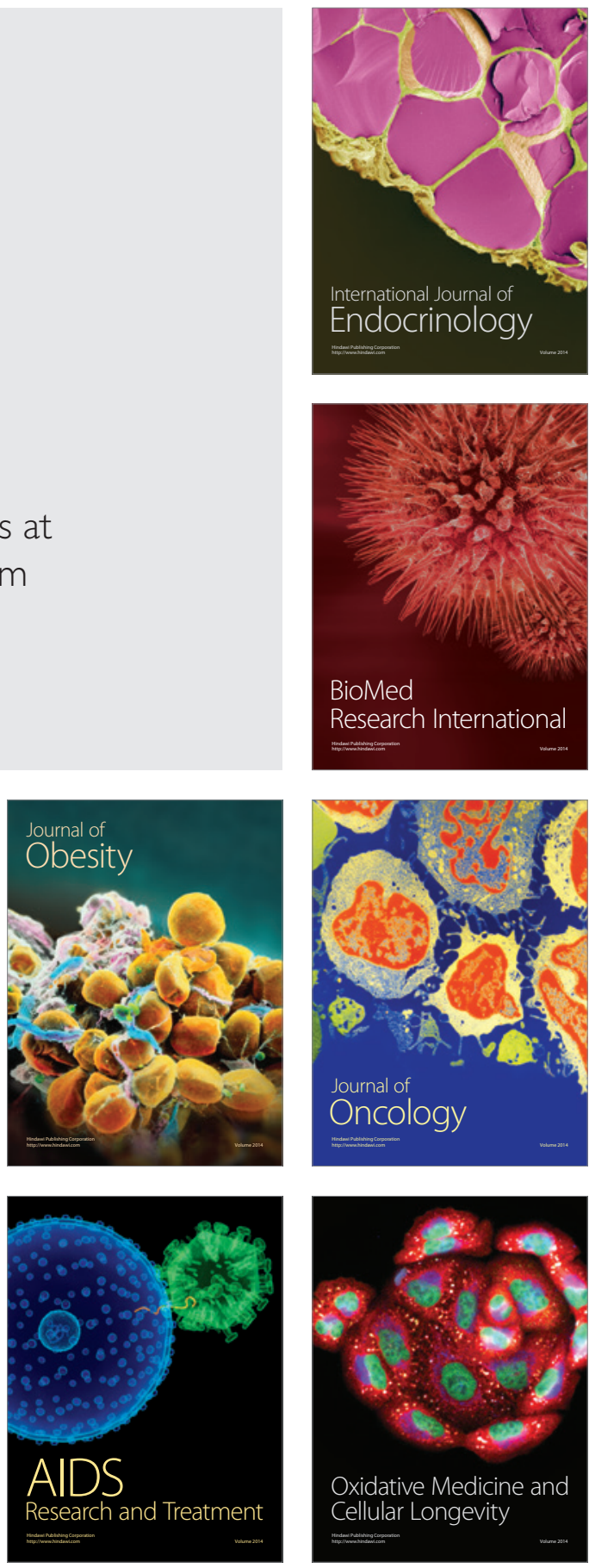